\title{
Évaluation de l'impact de la culture de colza sur la biodiversité aviaire en France : enjeux, méthodes, premiers résultats
}

\author{
Christophe SAUSSE ${ }^{1}$ \\ Eve PENILLARD ${ }^{1}$ \\ Aude BARBOTTIN ${ }^{2}$ \\ Frédéric JIGUET ${ }^{3}$ \\ ${ }^{1}$ Cetiom, Centre de Grignon, \\ avenue Lucien Brétignières, \\ 78850 Thiverval-Grignon, \\ France \\ <sausse@cetiom.fr> \\ 2 Inra, UMR 1048 SAD-APT, \\ BP01, \\ 78850 Thiverval-Grignon, \\ France \\ 3 Muséum National d'Histoire Naturelle, \\ CRBPO, BP 51, \\ 55 rue Buffon, \\ 75005 Paris, \\ France
}

L'impact du développement des biocarburants sur l'environnement fait l'objet de vifs débats en France et en Europe. Les préoccupations ont tourné jusque récemment autour de la capacité des biocarburants à limiter le changement climatique, mais leur impact sur la biodiversité est aujourd'hui questionné. Le sujet est rendu particulièrement complexe par le flou entourant le concept de biodiversité, sur lequel des acteurs variés projettent des représentations pas toujours bien assurées ni cohérentes entre elles (Le Guyader, 2008).

Sans vouloir clore le débat, cet article propose une réflexion sur le sens de cette question, et une méthode pour y répondre, en distinguant ce qui relève des indicateurs, du cadre théorique pour le diagnostic et des données mobilisables. Nous proposons d'utiliser des indices de biodiversité aviaire, et distin-

\begin{abstract}
Indices based on common birds are suitable to represent biodiversity, and are now commonly used. Farmland birds' indices in France and Europe show worrying trends. A diagnosis of the impact of oilseed crops raises methodological issues. Ecological studies usually establish statistical links between common birds and agriculture described by great types of farming systems. The specific case of landscapes with cereals cropping systems requires more precise descriptions. We carried out a study using national databases to assess the impact of land use at the scale of small agricultural regions. The importance of oilseed rape area was not a major determinant of birds' indices, and the statistical models explained little of the observed variability. This suggests the diagnosis should take other factors into account, like management of the cropping systems and the landscape configuration. This exploratory study based on statistical correlations is complementary to analytical and accurate field studies more suitable to highlight causality effects.
\end{abstract}

Key words: oilseed rape, biodiversity, land use, farmland birds

guons trois niveaux d'analyse correspondant au cadre de l'écologie du paysage et à certains leviers mobilisables par les acteurs de la filière des oléagineux : I'utilisation du territoire, l'aménagement du paysage et la gestion des systèmes de culture. Une étude préliminaire centrée sur le colza a permis d'aborder le niveau de l'utilisation du territoire. Nous en présentons les principaux résultats, avant d'aborder des perspectives d'approfondissement sur les deux autres niveaux.

\section{Pourquoi les oiseaux ?}

\section{Intérêt des indicateurs de biodiversité aviaire}

La biodiversité renvoie à la diversité des espèces et des écosystèmes ainsi qu'à la diversité génétique. Décrire I'ensemble de la biodiversité est impossible. Si I'on s'attache à la diversité des espèces, la plus médiatisée, celle-ci doit être estimée à partir de mesures sur une ou plusieurs espèces. Les informations recueillies sur une seule espèce peuvent donner lieu à des inférences abusives: l'espèce est corrélée à la biodiversité totale ou bien ce qui est bon pour une espèce l'est pour toutes. Travailler sur un groupe d'espèces diminue ce risque, mais présente l'inconvénient de compliquer la réalisation et I'interprétation du diagnostic : les traits démographiques d'une espèce peuvent être mis en relation avec des caractéristiques de milieux. Chaque espèce ayant des traits démographiques particuliers, les grouper rendra moins lisibles de tels liens de cause à effet. Nous sommes ici confrontés à un dilemme entre valeur heuristique de l'indice (i.e. sa capacité à être relié à des pratiques de

Pour citer cet article : Sausse C, Penillard E, Barbottin A, Jiguet F. Évaluation de l'impact de la culture de colza sur la biodiversité aviaire en France : enjeux, méthodes, premiers résultats. OCL 2011 ; 18(3) : 145-52. doi : 10.1684/ocl.2011.0379 
gestion ou bien à produire un modèle de recherche) et représentativité (i.e. corrélation avec la biodiversité totale). Sa résolution impose de bien clarifier I'objectif poursuivi. La question posée est celle de l'impact des oléagineux sur la biodiversité dans son ensemble, que nous cherchons à protéger en raison de sa valeur intrinsèque. Il ne s'agit donc pas de choisir un indicateur ad hoc en vue d'un but particulier. Le critère prioritaire est la représentativité.

Les oiseaux sont considérés comme de bons candidats pour l'élaboration d'indices représentatifs de la biodiversité, car ils sont globalement au sommet de la pyramide alimentaire et dépendent du bon fonctionnement de toute la chaîne trophique. Ainsi, le Farmland Bird Index basé sur les abondances des oiseaux nicheurs spécialistes des espaces agricoles permet de rendre compte de la biodiversité dans ce type d'espace (Gregory et al., 2005). II est aujourd'hui un des indicateurs structurels du développement durable au niveau de I'Union européenne, et peut être adapté pour piloter des politiques nationales, à l'exemple de la Suisse (Birrer et al., 2011). Le calcul d'indices de biodiversité aviaire est rendu possible par l'existence de réseaux d'observation produisant des données offrant une bonne couverture nationale, comme le Suivi temporel des oiseaux communs (STOC) en France. Ces bases de données, outre leur rôle pour le suivi et l'évaluation de politiques publiques, rendent possible la réalisation d'études d'impact. Enfin, les oiseaux ont l'avantage d'être visibles. Souvent appréciés pour leur valeur culturelle et esthétique, parfois redoutés, ils ne laissent pas indifférents le grand public, dont les agriculteurs font partie.

\section{Le statut des oiseaux spécialistes des milieux agricoles se dégrade}

Plusieurs études basées sur le Farmland Bird Index ont montré le déclin des populations d'oiseaux spécialistes des espaces agricoles au niveau national (Julliard et Jiguet, 2005) et européen (Donald et al., 2000, 2006). L'analyse des causes de ce déclin est basée principalement sur des corrélations statistiques entre indices de biodiversité et variables descriptives de l'agriculture.

Des études ont été menées à l'échelon national. Ainsi, Donald et al. (2000) ont mis en cause le rôle négatif sur le Farmland Bird Index de "I'intensification " des agricultures européennes, qu'ils ont caractérisées, pour les systèmes de grandes cultures, par des niveaux de rendement moyens en céréales pour chaque pays. Scholefield et al. (2011) ont montré pour la France une corrélation positive entre superficie en tournesol et Farmland Bird Index entre 1990 et 2007, et en déduisent qu'une augmentation des surfaces de cette culture sera mécaniquement bénéfique. Cette conclusion soulève plus de questions qu'elle n'en résout. Vues les superficies en cause, il est improbable que la diminution des superficies en tournesol les deux dernières décennies ait été la cause de la diminution des populations d'oiseaux. Une confusion d'effet avec d'autres évolutions structurelles des paysages agricoles est plus probable. L'établissement de corrélations à l'échelle locale (quelques $\mathrm{km}^{2}$ autour des points d'observation) permet d'affiner ces constats globaux. En enquêtant sur 25 paysages de $16 \mathrm{~km}^{2}$ dans différents pays européens, Billeter et al. (2008) ont montré que l'azote par ha de SAU était le second facteur prédictif de la richesse spécifique de l'avifaune, après la proportion d'éléments semi-naturels. L'interprétation de cette dernière variable est possible dans le cadre théorique de l'écologie du paysage (cf. infra), mais que signifie une dose d'azote par ha pour une population d'oiseaux ? L'établissement de liens de causalité semble ici beaucoup plus délicat, et là aussi, une confusion d'effet est possible. Certaines études mobilisent des bases de données nationales pour multiplier le nombre de paysages étudiés. Les variables de description de l'agriculture sont dans ce cas agrégées et peu détaillées (e.g. qualification en Haute Valeur Naturelle, Doxa et al., 2010), ou se réfèrent à des concepts de l'écologie comme la fragmentation et la perturbation des habitats (Devictor et al., 2007).

En sus de leur intérêt strictement scientifique, ces approches holistiques peuvent fournir des informations utiles pour orienter des politiques agricoles, par exemple des changements de systèmes de production ou la promotion de l'agriculture biologique. Des études sont en cours pour les coupler avec des modèles économiques en vue d'établir des scénarios de co-viabilité entre agriculture et biodiversité (Mouysset et al., 2011). Toutefois, ces approches ne sont pas assez détaillées pour travailler à I'amélioration des pratiques dans le cadre d'un système de production donné (Hole et al., 2005), ce qui est l'objectif de la plupart des organismes de développement travaillant au contact des agriculteurs. Autrement dit, ces études ne permettent pas à un institut technique agricole d'élaborer du conseil.

À l'opposé de ces approches holistiques, les naturalistes ont développé un corpus de connaissances sur les particularités de chaque espèce, qui peuvent éclairer dans une certaine mesure les corrélations observées à des échelles englobantes. Par exemple, il a été démontré que les populations d'alouettes des champs sont sensibles au mode de gestion des intercultures qui fournissent des ressources alimentaires à une période critique pour ces oiseaux (Donald et al., 2001). Ces connaissances peuvent déboucher sur des propositions de pratiques de gestion (voir par exemple, toujours pour l'alouette des champs, Morris et al., 2004). L'inconvénient de ces approches est leur caractère local et mono-espèce.

Les oiseaux donnant lieu au calcul $d^{\prime}$ indicateurs reconnus et représentatifs de la biodiversité, une connaissance fine de l'impact des activités agricoles permettrait d'orienter les actions à mener et de les évaluer. Pour un institut technique comme le Cetiom, cela signifie travailler sur variables permettant d'isoler un éventuel effet "oléagineux " correspondant à des leviers mobilisables par les acteurs de la filière. L'exercice peut sembler difficile car les populations d'oiseaux sont soumises à des facteurs agissant à de vastes échelles de temps et d'espace. Peut-on isoler un effet " oléagineux " en évitant les confusions d'effets évoquées plus haut ? Avant de répondre, il convient de préciser le cadre théorique qui sera utilisé pour décrire les objets en question.

\section{L'écologie du paysage comme cadre d'analyse}

Le diagnostic peut être fondé sur la notion $d$ 'habitat. Les populations d'oiseaux se nourrissent et se reproduisent dans des habitats spécifiques. Ces derniers peuvent être de qualité variable, plus ou moins aptes à fournir des ressources alimentaires au bon moment et à offrir abris et espace de nidification. 
La théorie de l'écologie des paysages soutient que la biodiversité peut être mise en relation avec les caractéristiques du paysage conçu comme un système formé de divers habitats. Dans une revue des effets des mosaïques paysagères sur la biodiversité, Bennett et al. (2006) identifient trois composantes des paysages pouvant influer sur la biodiversité : l'étendue de l'habitat favorable à l'espèce (ou groupe) considérée, la composition ou part de chacun des habitats dans la mosaïque et la configuration spatiale de ces habitats qui prend en compte leur connectivité. À une échelle englobante, la variation de biodiversité entre paysages peut être attribuée à des facteurs bio-géographiques: les espèces ont des aires de répartition propres.

L'intégration des " grandes cultures " à ce cadre théorique est en cours. Les travaux en écologie ont traditionnellement assimilé les grandes cultures au mieux à un habitat unique, au pire à une " matrice ", c'est-à-dire une sorte de no man's land entre habitats d'intérêt (l'exemple type étant des îlots forestiers). Cette vision évolue. Les paysages anthropisés sont aujourd'hui considérés comme générateurs d'une biodiversité tout aussi valable que la biodiversité présente dans des milieux naturels, pour la simple raison que ces derniers sont réduits en Europe à la portion congrue. Les concepts de l'agronomie, comme la parcelle et le système de culture, trouvent aujourd'hui leur place dans les travaux menés en écologie du paysage.

La parcelle cultivée forme un habitat caractérisé par d'importantes perturbations: travail du sol, succession des cultures, exportation des récoltes... À proprement parler, chaque culture et type d'interculture constitue un habitat plus ou moins favorable, et l'hétérogénéité de ces habitats, auxquels il faut ajouter des éléments périphériques (bordures, haies...), contribue à la qualité du grand type d'habitat formé par les grandes cultures. Cette hétérogénéité peut être abordée au niveau spatial (taille et forme des parcelles, mosaïque de cultures une année donnée), mais aussi temporelle. En effet, la diversité des taxons inféodés à une parcelle dépend non seulement de l'état présent de cette parcelle, mais aussi de son histoire, en d'autres termes de la gestion du système de culture. Par ailleurs, les états successifs des parcelles dans le paysage provoquent une modification de sa structure (Baudry et al., 2003).

Ce cadre permet d'analyser les facteurs affectant la biodiversité aviaire en paysage de grandes cultures selon trois niveaux, qu'il est possible de relier à des leviers d'action: utilisation du territoire, aménagement du paysage et gestion des systèmes de culture (tableau 1). Ce cadre plaide pour une démarche systémique prenant en compte les trois niveaux, et leur possible interaction comme le suggèrent Tscharnke et al. (2005). Le diagnostic doit d'abord hiérarchiser la contribution de chaque niveau, pour éviter les fausses pistes avant toute tentative d'approfondissement. Par exemple, identifier les pratiques culturales faisant varier les ressources alimentaires consommées par les oiseaux sur les parcelles (qualité d'habitat) peut aboutir à une déduction erronée: sans analyse au niveau du paysage, il n'est pas possible de prédire en quoi des variations de pratiques sur les parcelles contribueront aux ressources totales. Autrement dit, si les populations d'oiseaux sont surdéterminées par la composition ou la configuration des habitats, alors travailler sur leur qualité aura une utilité réduite. Bien entendu, ce raisonnement ne préjuge en rien des résultats du diagnostic, qui pourront tout aussi bien montrer une contribution importante des traitements phytosanitaires ou du travail du sol! L'idée est simplement de concevoir judicieusement le diagnostic en vue d'établir des priorités d'action.

\section{La question des données}

Le cadre d'analyse étant posé, quelles données mobiliser pour relier état de la biodiversité aviaire et variables explicatives? Les données sur la biodiversité aviaire existent grâce aux réseaux d'ornithologues volontaires, ou peuvent être acquises localement. La description des paysages agricoles (aux trois niveaux) est plus difficile. Une première solution consiste à recueillir des données détaillées sur quelques paysages. Cette méthode présente l'avantage de rendre possible la production de modèles explicatifs, grâce au niveau de détail élevé des variables descriptives des milieux. Mais ces modèles resteront locaux, c'est-à-dire que se posera la question de leur extrapolation à d'autres paysages et régions. Une seconde solution consiste à partir de bases de données nationales, moins détaillées, mais offrant la possibilité de travailler sur un grand nombre de situations. L'avantage est une meilleure généralisation, mais qui se paye d'une moindre valeur explicative.

Ces deux approches peuvent être combinées. C'est le cas dans le projet FARMBIRD associant INRA et CNRS (financement ANR), qui combine enquêtes de terrain et travail à partir de bases de données descriptives des systèmes de production. Pour rester complémentaires des travaux réalisés

Tableau 1. Les différents niveaux d'analyse de la biodiversité aviaire (d'après Bennett et al., 2006).

\begin{tabular}{|lll|}
\hline Niveau d'analyse & Action possible & Levier/filière oléagineux \\
\hline 0. biogéographie & $/$ & $/$ \\
\hline $\begin{array}{l}\text { 1. étendue \& composition } \\
\text { des habitats }\end{array}$ & $\begin{array}{l}\text { Utilisation du territoire : Assolement, } \\
\text { développement de filière }\end{array}$ & $\begin{array}{l}\text { Faire varier le \% d'oléagineux } \\
\text { dans la sole }\end{array}$ \\
\hline 2. configuration des habitats & $\begin{array}{l}\text { Aménagement du paysage : infrastructures } \\
\text { agro-écologiques (bandes enherbées, haie...), } \\
\text { taille des parcelles, mosaïque de cultures }\end{array}$ & $\begin{array}{l}\text { Aménager les parcelles (concerne toute } \\
\text { les filières); éviter la constitution de gros blocs } \\
\text { de culture }\end{array}$ \\
\hline 3. qualité des habitats & $\begin{array}{l}\text { Gestion des systèmes de culture : pratiques } \\
\text { culturales, successions, gestion des intercultures }\end{array}$ & Adapter les pratiques sur oléagineux \\
\hline
\end{tabular}




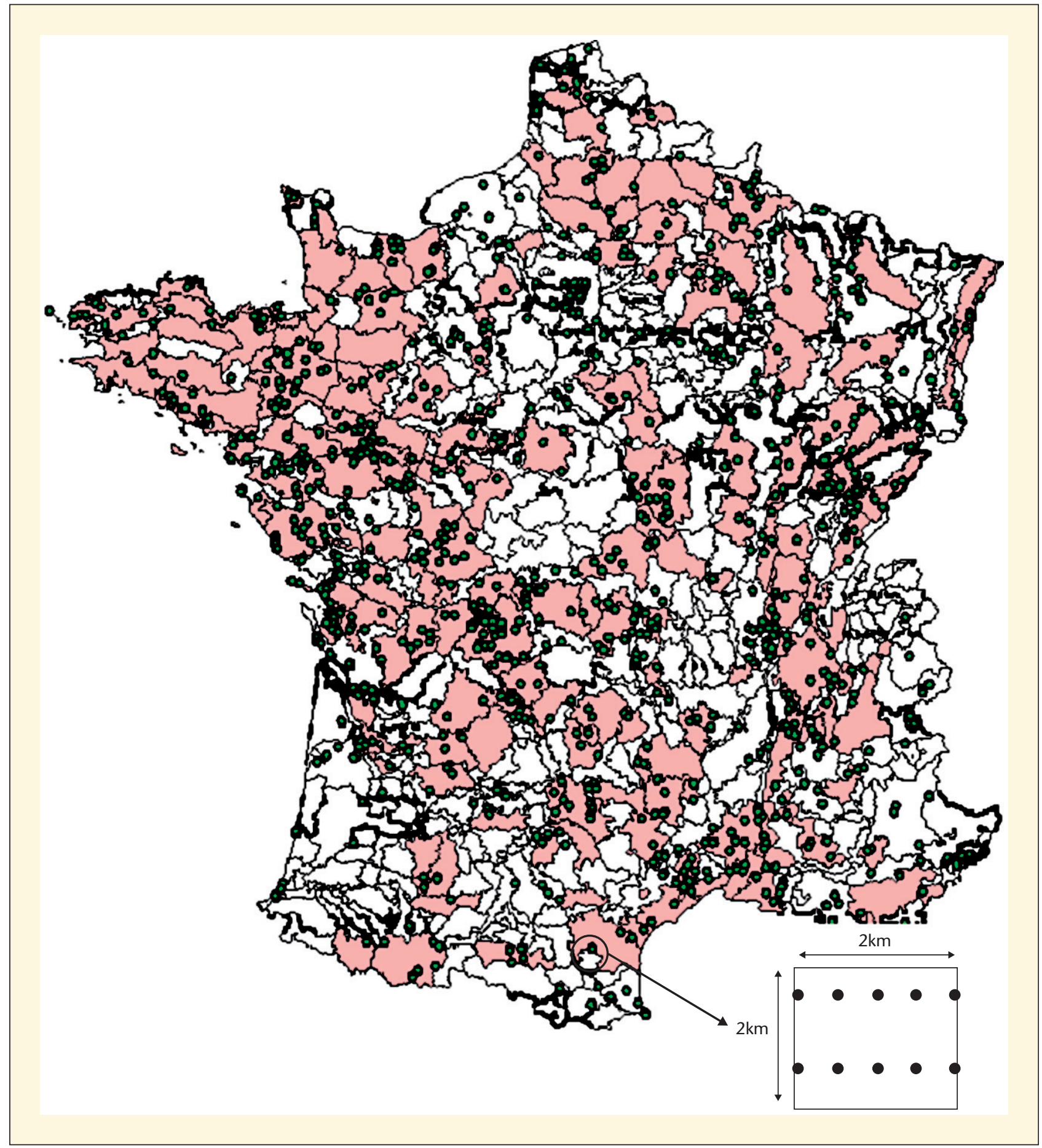

Figure 1. Le réseau STOC comprenait, en 2004, 902 carrés contenant chacun 10 points. Les PRA en rose sont celles retenues pour l'étude.

dans le cadre de ce projet, nous avons opté pour l'exploitation de bases de données nationales, en nous concentrant sur la description des grandes cultures. Un premier travail réalisé en 2010 a permis de donner des éléments de réponse à la question de l'impact de I'utilisation du territoire (tableau 1, niveau 1). Cette étude était exploratoire et conçue comme un test de méthode. Nous en présentons ici les résultats, et discutons des perspectives d'approfondissement pour intégrer dans le diagnostic les niveaux de l'aménagement du paysage et de la gestion des systèmes de culture.
Impact de I'utilisation du territoire : premiers résultats

Matériel et méthodes

Nous avons tiré parti de bases de données existantes pour analyser la 
relation entre l'usage du territoire et des indicateurs de biodiversité aviaire fournis par le programme STOC. Ce programme agrège des données provenant d'un réseau d'ornithologues volontaires. Des carrés de $2 \times 2 \mathrm{~km}$ sont tirés aléatoirement autour des communes des observateurs. Chaque carré est suivi 2 fois par an. Les oiseaux observés et entendus sont dénombrés sur 10 points par carré (figure 1).

Le réseau incluait 902 carrés en 2004, correspondant à l'année d'étude. Nous avons sélectionné 415 carrés incluant au moins 5 points déclarés par les observateurs comme espaces agricoles. Nous avons calculé 4 indicateurs :

- I'abondance des oiseaux agricoles communs. Nous sommes partis d'une liste de 18 espèces, correspondant aux 20 prises en compte dans le Farmland Bird Index, moins 2 connues pour induire une forte variabilité locale (le corbeau freu et le faisan). Nous avons divisé cette liste en deux sous-groupes: les spécialistes des espaces ouverts comme l'alouette et les spécialistes des espaces semiouverts et fermés (par exemple la huppe fasciée), conformément au classement opéré par les naturalistes du Centre de recherche par le baguage des populations d'oiseaux (CRBPO);

- la richesse spécifique. Cet indice correspond au nombre total d'espèces par carré ;

- I'indice de spécialisation de la communauté (CSI). L'indice de spécialisation $d^{\prime}$ 'une espèce (SSI) est égal au coefficient de variation de son abondance relative au sein de 18 habitats. Cet indice est utilisé pour distinguer les espèces spécialistes observées dans un habitat particulier avec de fortes valeurs de SSI (e.g. SSI de l'alouette des champs $=1,16)$, et les espèces plus généralistes observées dans une plus large gamme d'habitats, caractérisées par un SSI plus faible. Le CSI moyen par point est la moyenne des abondances relatives de chaque espèce pondérées par leur SSI. Le CSI d'un carré est égal à la moyenne des CSI sur les 10 points ;

- I'indice trophique de la communauté (CTI) reflétant la diversité des régimes alimentaires. Des références existent sur les pourcentages de plantes, d'invertébrés et de vertébrés dans le régime de chaque espèce. L'indice trophique de l'espèce est la somme des trois pourcentages pondérée par 1 pour les plantes, 2 pour les invertébrés, 3 pour les vertébrés. Ainsi, une espèce au sommet de la chaine alimentaire aura un TTI supérieur à celui d'une espèce herbivore (e.g. TTI de la buse variable = 2,9 vs. TTI de l'alouette des champs = 1,3). Le CTI par point correspond à l'abondance moyenne de chaque espèce divisée par son TTI. Le CTI par carré est la valeur moyenne des 10 points. Cet indicateur reste expérimental et fait encore l'objet de tests.

L'usage des territoires a été décrit à partir de données Corine Land Cover et de données statistiques de I'ONIGC (Office national interprofessionnel des grandes cultures). Nous avons choisi de travailler à l'échelle des petites régions agricoles (PRA), qui rendent bien compte de la diversité géographique des systèmes de production (Mignolet, 2008). Les PRA agrègent des communes, alors que les données statistiques ne sont disponibles qu'à l'échelon du canton. Nous avons choisi la règle suivante pour changer d'échelle : une PRA est décrite à partir des cantons qui y sont strictement inclus, i.e. qui ne sont pas à cheval sur plusieurs PRA. L'étude a couvert 182 PRA du territoire national
Tableau 2. Données utilisées pour l'élaboration des modèles d'analyse de la biodiversité aviaire

\begin{tabular}{|ll|}
\hline Données & Source \\
\hline $\begin{array}{l}\text { Surfaces en colza, } \\
\text { céréales, jachère } \\
\text { agronomique }\end{array}$ & $\begin{array}{l}\text { ONIGC sur la base } \\
\text { des déclarations } \\
\text { PAC }\end{array}$ \\
\hline $\begin{array}{l}\text { Surfaces en prairies } \\
\text { et espaces } \\
\text { semi-naturels }\end{array}$ & Corine Land Cover \\
\hline SAU & Agreste (RGA 2000) \\
\hline $\begin{array}{l}\text { Surface des } \\
\text { communes }\end{array}$ & IGN \\
\hline
\end{tabular}

contenant au moins un carré STOC en milieu agricole, chaque PRA étant décrite avec les variables : pourcentage de colza, de céréales, de jachère agronomique, de prairie et d'espaces seminaturels dans la superficie totale de la PRA ou la SAU (tableau 2).

Les relations entre indices de biodiversité aviaire sur les 415 carrés STOC et usage du territoire sur les 182 PRA ont été analysées par régression linéaire multiple (modèle log-linéaire pour les abondances, linéaire pour les CSI, CTI et richesse spécifique). Les modèles ont été sélectionnés grâce au critère d'information d'Akaike.

\section{Résultats et discussion}

Le tableau 3 donne les corrélations entre variables explicatives. Ces corrélations doivent être interprétées avec précaution en raison de possibles relations non linéaires. Le tableau 4 donne pour chaque modèle de régression l'estimation des coefficients et la variabilité expliquée par le modèle. Les surfaces sont ici rapportées à la surface totale des PRA. Les surfaces

Tableau 3. Matrice de corrélation entre variables explicatives

\begin{tabular}{|c|c|c|c|c|c|}
\hline & Colza & Céréales & Jachère & Prairies & $\begin{array}{l}\text { Espaces semi- } \\
\text { naturels }\end{array}$ \\
\hline Colza & 1 & & & & \\
\hline Céréales & 0,61 & 1 & & & \\
\hline Jachère & 0,55 & 0,74 & 1 & & \\
\hline Prairies & $-0,27$ & $-0,41$ & $-0,44$ & 1 & \\
\hline Espaces semi-naturels & $-0,33$ & $-0,73$ & $-0,62$ & 0,72 & 1 \\
\hline
\end{tabular}


Tableau 4. Estimation des coefficients des modèles de régression multiples liant indices de biodiversité aviaire et utilisation du territoire exprimée en pourcentage de surface de la petite région agricole. $X$ indique des variables non retenues.

\begin{tabular}{|c|c|c|c|c|c|c|c|}
\hline Indice & Modèle & \%Colza & \%Céréales & \%Prairie & \%Jachère & $\begin{array}{l}\text { \%Semi- } \\
\text { naturel }\end{array}$ & $\begin{array}{l}\text { Variabilité } \\
\text { expliquée }\end{array}$ \\
\hline $\begin{array}{l}\text { Abondance des spécialistes } \\
\text { agricoles } \\
\text { moyenne }=40,1\end{array}$ & Log-linéaire & 0,047 & 0,021 & $x$ & $-0,076$ & 0,002 & $13,9 \%$ \\
\hline $\begin{array}{l}\text { Abondance des spécialistes } \\
\text { des espaces ouverts } \\
\text { moyenne }=16,7\end{array}$ & Log-linéaire & 0,058 & 0,038 & $-0,008$ & $-0,075$ & 0,004 & $28,0 \%$ \\
\hline $\begin{array}{l}\text { Abondance des spécialistes } \\
\text { des espaces semi-ouverts } \\
\text { et fermés } \\
\text { moyenne }=23,3\end{array}$ & Log-linéaire & 0,035 & 0,006 & 0,006 & $-0,061$ & $x$ & $4,0 \%$ \\
\hline $\begin{array}{l}\mathrm{CTI} \\
\text { moyenne }=1,6\end{array}$ & Linéaire & $-0,004$ & $-0,002$ & $x$ & $x$ & $x$ & $16,3 \%$ \\
\hline $\begin{array}{l}\text { CSI } \\
\text { moyenne =0,66 }\end{array}$ & Linéaire & 0,010 & $x$ & $-0,004$ & $-0,022$ & $x$ & $13,8 \%$ \\
\hline $\begin{array}{l}\text { Richesse spécifique } \\
\text { moyenne }=49,0\end{array}$ & Linéaire & $x$ & $-0,184$ & 0,107 & 2,55 & $x$ & $3,5 \%$ \\
\hline
\end{tabular}

rapportées à la SAU génèrent des résultats sensiblement identiques.

Les pourcentages de surface en colza sont positivement reliés aux abondances et au CSI, mais négativement au CTI.

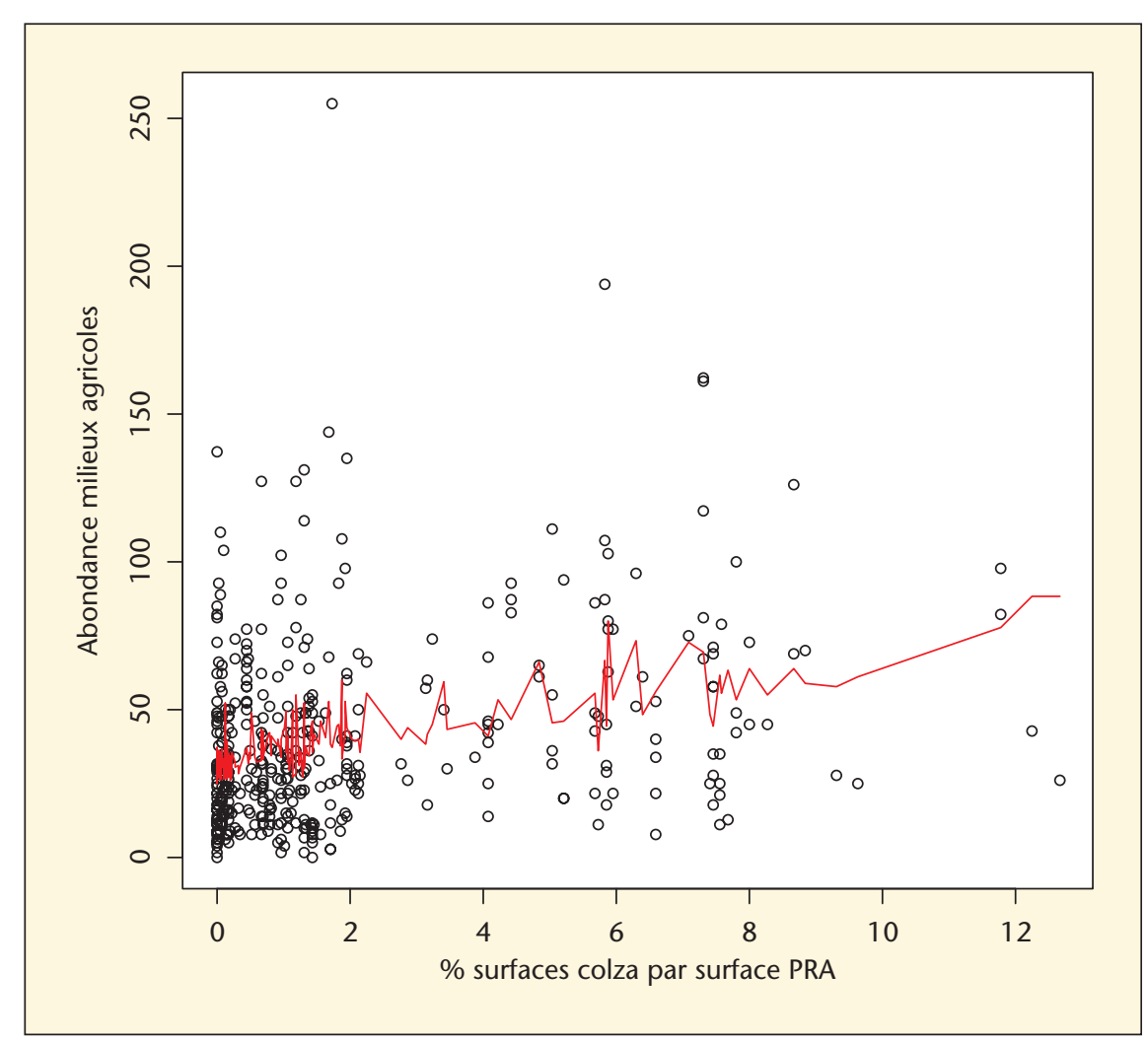

Figure 2. Abondance des spécialistes des milieux agricoles en fonction de la surface en colza dans la PRA. La ligne rouge représente les valeurs prédites par le modèle complet incluant toutes les variables.

Mais ces liens sont à peu près identiques en ce qui concerne les céréales. Les surfaces en céréales et en colza étant corrélées, cela suggère un impact de l'étendue des grandes cultures globale- ment favorables à une avifaune spécialisée, sans possibilité de déterminer un effet propre au colza.

Bien que la jachère agronomique soit positivement corrélée aux céréales et au colza, elle est négativement corrélée aux indicateurs de biodiversité aviaire, à l'exception de la richesse spécifique. II n'est pas possible d'identifier les mécanismes sous-jacents à ces relations statistiques : la jachère agronomique estelle réellement un habitat défavorable (hypothèse de ressources alimentaires limitées par un couvert permanent), ce qui contredit l'opinion commune? Ou bien y-a-t-il confusion d'effet avec $d^{\prime}$ autres facteurs non pris en compte dans I'analyse ? Cet exemple illustre les limites de cette approche englobante pour analyser en profondeur les mécanismes de cause à effet.

Le tableau 4 montre que la variabilité des indicateurs de biodiversité aviaire est faiblement expliquée par les variables sélectionnées. Par exemple, I'abondance des spécialistes agricoles dans les PRA contenant de 7 à $8 \%$ de colza varient entre 11 et 162 (figure 2). Ces résultats montrent que la superficie de colza dans un paysage donné n'est pas un facteur explicatif majeur et ne menace pas en elle-même la biodiversité aviaire. En conséquence, le diagnostic doit prendre en compte d'autres facteurs en vue d'identifier ultérieurement des stratégies opérationnelles d'amélioration de la biodiversité. 
À l'issue de cette étude exploratoire, nous n'avons pas pu répondre complètement à la question de l'impact de I'utilisation du territoire. En effet, la méthode et les données mobilisées ont imposé plusieurs limites.

Les résultats sont issus de comparaisons pour une année donnée. Une approche diachronique, comparant des évolutions de biodiversité aviaire dans des PRA ayant connu des évolutions contrastées des superficies en colza, aurait-elle pu donner une conclusion différente? Autrement dit, l'introduction de colza dans une PRA modifie-t-elle, dans un sens ou dans l'autre, la baisse tendancielle des indices de biodiversité aviaire ? La question reste ouverte et en appelle une autre : l'introduction du colza implique une modification des systèmes de culture, et c'est peut-être sur la répartition des systèmes de culture dans le territoire et leur évolution que doit porter l'analyse, et non sur le seul assolement. Cette approche est à même de rendre compte du poids de I'histoire dans la biodiversité observée une année donnée.

Un autre problème à résoudre est celui de l'échelle la plus adaptée au diagnostic et aux mesures correctives. Les résultats mitigés obtenues à l'échelle des PRA suggèrent qu'elle n'est pas la plus pertinente, c'est-à-dire celle générant le meilleur coefficient de détermination des modèles d'analyse. Nous manquons d'éléments pour déterminer cette échelle a priori : la question de l'espace nécessaire à une population d'oiseaux nicheurs pour vivre et se reproduire $n^{\prime}$ est pas résolue. Des analyses complémentaires réalisées à l'échelle des cantons, que nous ne détaillerons pas ici, $n^{\prime}$ ont pas amélioré les performances des modèles de manière significative. Les résultats suggèrent de poursuivre les analyses à des échelles encore plus resserrées, ce qui suppose de disposer de données idoines.

Ces obstacles ne sont pas insurmontables. L'exploitation du registre parcellaire graphique (RPG) permet de les franchir: I'occupation des îlots sur plusieurs années permet en effet de décrire les systèmes de culture sur n'importe quel paysage de grandes cultures, d'un îlot jusqu'à la France entière. Ainsi, il est en principe possible de travailler sur des spots de taille variée autour des carrés STOC et sur plusieurs années.

\section{Perspectives pour la prise en compte de I'aménagement des paysages et de la gestion des systèmes de culture}

Vues les faibles performances des modèles statistiques, l'étude préliminaire montre que le diagnostic ne saurait se limiter à l'impact de I'utilisation du territoire. II convient de vérifier si la prise en compte des deux autres niveaux de l'aménagement du paysage et de la gestion des systèmes de culture améliore le diagnostic. Mais la méthode se heurte à un problème pratique : les bases de données nationales sont-elles assez détaillées pour travailler à ces niveaux ?

Les cartes issues du RPG offrent I'opportunité de travailler sur la structuration de la mosaïque de culture. La prise en compte d'infrastructures agro-écologiques (bandes enherbées, haies, talus) semble en revanche plus difficile. Les données Corine Land Cover sont assez peu détaillées, mais les objectifs $\mathrm{d}^{\prime}$ " éléments topographiques " conditionnant les aides Européennes offrent à terme l'opportunité de disposer de données plus détaillées en zones de grandes cultures. Dans l'immédiat, les études poussées sur de petits territoires sont les plus prometteuses. Quant aux pratiques culturales, l'exploitation d'enquêtes sur des échantillons de parcelles pose des problèmes d'extrapolation. Mais plusieurs voies sont envisageables pour les résoudre, comme les géostatistiques et l'utilisation de co-variables (e.g. type de sol).

\section{Conclusion}

L'exploitation intensive des bases de données nationales permettra, nous l'espérons, de dépasser les constats globaux sur l'impact de l'agriculture "intensive " grâce à une analyse à plusieurs niveaux : utilisation du territoire, aménagement du paysage et gestion des systèmes de culture. Cette démarche basée sur des corrélations statistiques est complémentaire d'études de terrain, plus à même de mettre en évidence des mécanismes agroécologiques dont la connaissance permet d'éviter les chausse-trappes des confusions d'effets. Si les résultats sont probants, cette décomposition de la question de l'impact de l'agriculture permettra d'affiner à terme les stratégies de gestion de l'avifaune dans les territoires de grandes cultures. Une telle démarche systémique est à même d'expliciter et de relativiser la contribution des oléagineux.

II convient toutefois de se garder d'un optimisme excessif. Ce type d'étude traite de jeux de données "bruitées" c'est-à-dire entachées d'incertitudes (effet " observateur " pour les oiseaux, qualité des données de base et variance générée par les méthodes d'estimation pour les paysages et les pratiques, etc.). D'autre part, les travaux réalisés jusqu'à présent ont produit des modèles assez peu explicatifs, révélateurs de grandes tendances. Notre objectif est d'améliorer ces modèles dans un sens plus compréhensible pour un public agricole, et d'en améliorer les performances, tout en s'attendant à ce que leur variabilité résiduelle reste élevée.

Pour des agronomes, ces méthodes et types de résultats peuvent être assez déconcertants : La mauvaise interprétation d'une corrélation statistique ne risque-t-elle pas d'aboutir à des coups d'épée dans l'eau, fatigants mais inutiles, alors que l'ennemi véritable court toujours ? Peut-on appeler à des changements de pratiques dont le coût est certain, pour des résultats localement aléatoires? D’un autre côté, l'outil traditionnel des agronomes qu'est l'expérimentation factorielle $n^{\prime}$ est probablement pas adapté à ces nouveaux objets d'étude. La recherche du "toute chose égale par ailleurs " risque de nous faire passer à côté de facteurs et d'interactions complexes, inattendus, opérant à des échelles de temps et d'espace variées.

Dans le doute que faire? Comment gérer le hiatus entre une recherche mal assurée, et I'injonction d'agir ? II n'existe probablement pas de méthode parfaite pour comprendre des êtres qui se laissent aussi peu contrôler que les oiseaux. L'heure est à la créativité grâce à une collaboration accrue entre écologues et agronomes.

\section{RÉFÉRENCES}

Baudry J, Burel F, Aviron S, et al. Temporal variability of connectivity in agricultural landscapes: Do farming activities help? Landscape Ecology 2003 ; 18 : 303-14. 
Bennett AF, Radford JQ, Haslem A. Properties of land mosaics: Implications for nature conservation in agricultural environments. Biological Conservation 2006 ; 133 : 250-64.

Billeter R, Liira J, Bailey D, et al. Indicators for agriculture in agricultural landscapes: a panEuropean study. J Appl Ecol2008; 45 : 141-50.

Birrer S, Markus J, Zbiden N. Evolution des populations d'oiseaux nicheurs dans les zones agricoles de 1990 à 2009. Recherche Agronomique Suisse $2011 ; 2$ : 66-71.

Devictor V, Julliard R, Clavel J, Jiguet F, Lee A, Couvet $\mathrm{D}$. Functional biotic homogenization of bird communities in disturbed landscapes. Global Ecology and Biogeography $2007 ; 17$ : 252-61.

Donald PF, Buckingham DL, Moorcroft D, Muirhead LB, Evans AD, Kirby WB. Habitat use and diet of skylarks Alauda arvensis wintering on lowland farmland in southern Britain. J Appl Ecol 2001 ; 38 : 536-47.

Donald PF, Green RE, Heat MF. Agricultural intensification and the collapse of Europe's farmland bird populations. Proc $R$ Soc Lond $B$ $2000 ; 268: 25-9$.
Donald PF, Sanderson FJ, Burfield IJ, van Bommel FPJ. Further evidence of continentwide impacts of agricultural intensification on European farmland birds, 1990-2000. Agr Ecosyst Environ 2006 ; 116 : 189-96.

Doxa A, Bas Y, Paracchini ML, Pointereau P, Terres JM, Jiguet F. Low-intensity agriculture increases farmland bird abundances in France. J Appl Ecol 2010 ; 47 : 1348-56.

Gregory RD, van Strien A, Vorisek $\mathrm{P}$, et al. Developing indicators for European birds. Phil Trans R Soc 2005 ; 360 : 269-88.

Hole DG, Perkins AJ, Wilson JD, Alexander IH, Grice PV, Evans AD. Does organic farming benefit biodiversity? Biological Conservation $2005 ; 122$ : 113-30.

Julliard R, Jiguet $F$. Statut de conservation en 2003 des oiseaux communs nicheurs en France selon 15 ans de programme STOC. Alauda $2005 ; 73$ : 345-56.

Le Guyader H. La biodiversité : un concept flou ou une réalité scientifique? Courrier de I'environnement de I'INRA 2008 ; $\mathrm{n}^{\circ} 55$ : 7-26.

Mignolet C. Modélisation de I'organisation spatiale des systèmes agricoles et de son évolution dans des démarches d'appui au développement. Thèse AgroParisTech, $282 \mathrm{p}$.

Morris AJ, Holland JM, Smith B, Jones NE. Sustainable Arable Farming For an Improved Environment (SAFFIE) : managing winter wheat sward structure for Skylarks Alauda arvensis. Ibis 2004; 146 (Suppl. 2) : 155-62.

Mouysset L, Doyen L, Jiguet F, Allaire G, Léger F. Bio economic modeling for a sustainable management of biodiversity in agricultural lands. Ecological Economics 2011; [epub ahead of print]. doi :10.1016/j.ecolecon.2010.12.006.

Scholefield P, Firbank L, Butler S, Norris K, Jones LM, Petit $S$. Modelling the European Farmland Bird Indicator in response to forecast land-use change in Europe. Ecological Indicators 2011 ; 11: 46-51.

Tscharnke T, Klein AM, Kruess A, SteffanDewenter I, Thies C. Landscape perspectives on agricultural intensification and biodiversity - ecosystem service management. Ecology Letters $2005 ; 8: 857-74$. 